\title{
Lungenkrank im Flugzeug: Was ist zu beachten?
}

\author{
Wenn COPD-Patienten auf Reisen gehen, ist eine sorgfältige reisemedizinische Beratung \\ nötig. Das beginnt bereits bei den Vorbereitungen auf einen Flug, wie Prof. Dr. med. Adrian \\ Gillissen, Direktor der Klinik für Lungen- und Bronchialmedizin, Klinikum Kassel, erläutert.
}

\section{$?$}

Prof. Dr. med. Adrian Gillissen

Klinik für Lungen- und Bronchialmedizin, Klinikum Kassel

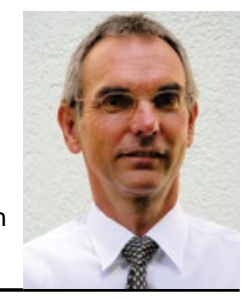

MMW: Können Asthma- und COPDSprays bei Flugreisen problemlos im Bordgepäck mitgenommen werden? Gillissen: Ja, das geht, da es sich um Medikamente handelt.

MMW: Der $\mathrm{O}_{2}$-Partialdruck in der Kabine entspricht dem auf 2500 Metern Meereshöhe. Was hat das für Konsequenzen für Lungenpatienten?

Gillissen: Nach Angaben der European Respiratory Society sollte der Sauerstoffdruck im Blut während eines Fluges mindestens $55 \mathrm{mmHg}$ betragen. Falls Patienten generell einen eher niedrigen Sauerstoffgehalt im Blut haben, kann es sein, dass der Wert im Flugzeug unter diese Schwelle sinkt. Dies kann auch passieren, wenn sie aufgrund einer akuten Verschlechterung während ihres Urlaubs, etwa aufgrund eines Infekts, den Rückflug antreten müssen.

\section{MMW: Wann ist ein COPD-Patient flugtauglich?}

Gillissen: In der Regel baucht ein COPDPatient, der in Ruhe normale Sauerstoffund Kohlendioxidwerte in der Blutgasanalyse aufweist, keine besonderen Vorsichtsmaßnahmen wegen des Sauerstoffdrucks in der Flugzeugkabine zu treffen. Aber: Vor Antritt einer Flugreise muss der Zustand der COPD stabil sein. Bei Zweifel an der Flugtauglichkeit sollte ein
Lungenfunktionstest und eine Blutgasanalyse durchgeführt werden. Man sollte auch darauf achten, dass die Patienten keine Ödeme haben.

\section{MMW: Wie ist das bei Emphysempati- enten?}

Gillissen: Leiden Patienten an einer COPD mit Lungenemphysem, sollte unbedingt im Vorfeld der Flugreise eine Untersuchung beim Lungenfacharzt erfolgen, der dann das Risiko für eine Flugreise abschätzen kann. Die Druckschwankungen in den Flugzeugkabinen kommerzieller Airlines prädisponieren aber offenbar nicht für eine erhöhte Pneumothoraxrate bei Emphysempatienten.

MMW: Worauf ist zu achten, wenn sauerstoffpflichtige Patienten fliegen wollen?

Gillissen: Die Mitnahme von flüssigem Sauerstoff ist auf Flugreisen grundsätzlich nicht gestattet. Gasdruckflaschen oder mobile Sauerstoffkonzentratoren sind oft erlaubt. Einige Fluggesellschaften schränken die Mitnahme von Konzentratoren jedoch auf bestimmte Modelle ein.

Auf der Homepage der Federal Aviation Administration findet sich eine Liste mit Empfehlungen für Fluggesellschaften mit geeigneten Geräten. Manche Fluggesellschaften bieten eine Sauerstoffversorgung an Bord an, die im Vorfeld gebucht werden muss. Dies kann aber teuer werden und die Krankenkassen übernehmen diese Kosten nicht notwendigerweise. Einige Fluggesellschaften verlangen ein ärztliches Attest, das die generelle Flugreisetauglichkeit feststellt. Tipps und Tricks für Sauerstoff-

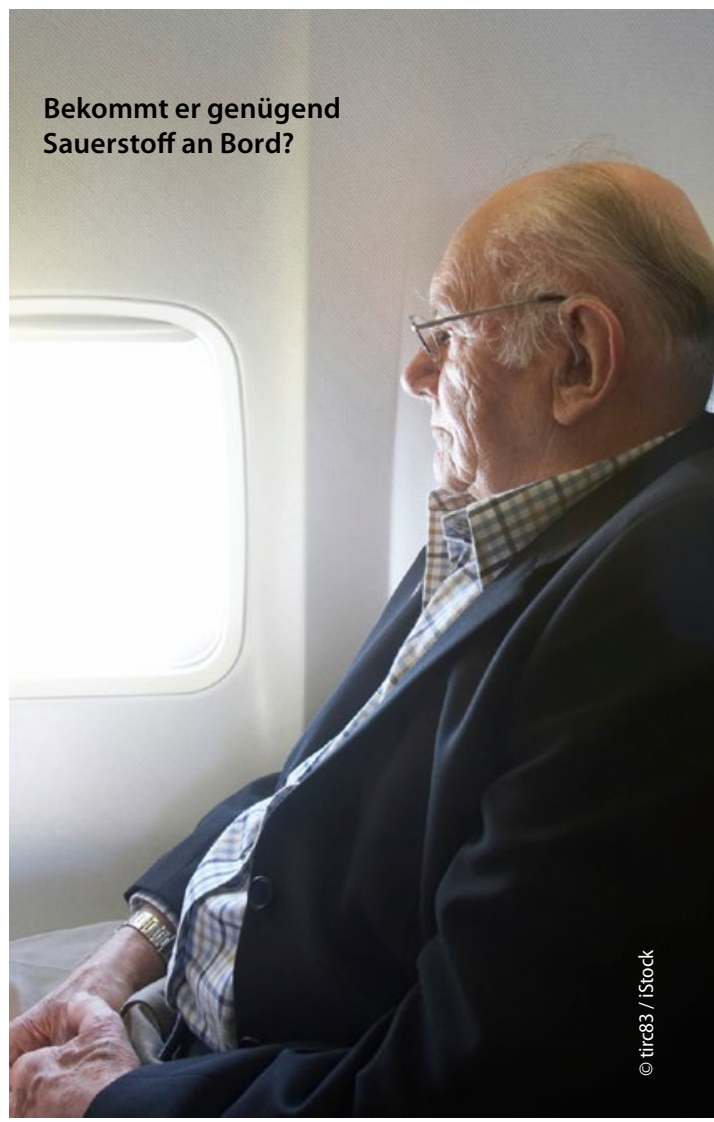

Patienten auf Reisen hat auch die Deutsche Sauerstoffliga (www.sauerstoffliga.de).

MMW: Kann die trockene Luft im Flugzeug Probleme bereiten?

Gillissen: In der Regel macht sie keine Probleme. Der Körper ist gut in der Lage, die Atemluft anzufeuchten. Man sollte aber beim Fliegen genug trinken, das gilt auch für Lungengesunde, denn bei niedriger Luftfeuchtigkeit wird über die Haut mehr Wasser an die Umgebungsluft abgegeben.

Interview: Dr. Beate Schumacher 